\title{
CASE OF CEREBRAL TUMOUR.
}

BY

A. HUGHES BENNETT, M.D., F.R.C.P., PHYGICIAN TO THE HOSPITAI FOR RPIIRPSY AND PARALYSIS, AND ASEISTANT PHYSICIAN TO THE WRSTMINETER HOSPITAL.

THE SORGIOAL TREATMENT

BY

RICKMAN J. GODLEE, M.S., F.R.C.S., SURGEON TO UNIVERSITY COLLEGE HOSPITAT.

Roceived Jaunary 18th-Read May 12th, 1885.

THE chief features of interest in the case, to which the attention of the Society is directed, are, that during life the existence of a tumour was diagnosed in the brain, and its situation localised, entirely by the signs and symptoms exhibited, without any external manifestations on the surface of the skull. This growth was removed without any immediate injurious effects on the intelligence and general condition of the patient. Although he died four weeks after the operation, the fatal termination was due, not to any special effects on the nervous centres, but to a secondary surgical complication. The case, moreover, teaches some important physiological, pathological, and clinical lessons, and suggests practical reflections which may prove useful to future medicine and surgery. 
History.-The patient was a farmer, æt. 25, who applied for advice to the Hospital for Epilepsy and Paralysis, Regent's Park, on November 3rd, 1884. His chief complaint was paralysis of the left hand and arm, which incapacitated him from work. He stated that his family history was unimportant, that he had always been temperate and in robust health, and that he never had suffered from syphilis or a day's illness of any kind in his life. About four years ago, while in Canada, a piece of timber fell from a house, struck him on the left side of the head and knocked him down. He thinks he lost consciousness for a few moments, after which he so far recovered as to be able to resume his work. On the following day he was quite well. With the exception of occasional slight headaches he afterwards remained in good health for a year, at the end of which time he first began to experience a feeling of twitching in the left side of his mouth and tongue. This soon developed into attacks of a paroxysmal character, which gradually became more pronounced and frequent, and continued to occur at irregular intervals. Some months afterwards he had a "fit" which began with a peculiar feeling in the left side of the face and tongue, and turning of the head to the left side. The sensation ran down the left side of the neck to the arm and leg, and culminated in loss of consciousness and general convulsions. For a few days subsequent to this the patient suffered from headache, and felt generally unwell, but ultimately regained his former condition. For two and a half years, although maintaining his robust health, he was subject to daily recurrences of the paroxysmal twitchings of the left side of the face without loss of consciousness, and also to the more severe general convulsive seizures with loss of consciousness, which occurred on an average about once a month. Six months before admission spasmodic twitchings of the left hand and arm, without loss of consciousness, were observed and these have continued daily, alternating with the already mentioned twitchings of the face, the two, however, rarely occurring at the same time. Shortly afterwards weakness 
of the left fingers, hand and forearm was experienced, which gradually increased to complete paralysis. Since the upper extremity began to be affected, there had been no recurrence of the general convulsive attacks with loss of consciousness. The patient was able to continue at work till August, 1884, when the weakness of the arm prevented him using his tools. Since then twitching of a like nature has taken place in the left leg, which usually supervenes upon, and is accompanied by, similar attacks in the arm on the same side. Quite recently the left lower extremity has been weak and the patient has walked a little lame.

Present condition.-On examination the patient was found in robust general health. His intelligence was unimpaired. All his organs and functions were normal except those about to be described. He suffered from frequent violent paroxysmal attacks of lancinating pain in the head, not localised but diffused over the vertex. There was nothing abnormal to be detected on the scalp or skull, and there was no special tenderness. On deep and hard pressure there was an area, not strictly defined, which seemed to be more sensitive than the neighbourhood. This was situated in the parietal region, close to the right of the sagittal suture, on a level with a line drawn vertically from the anterior portion of the external meatus of the ear. The movements of the eyeballs and pupils were normal ; vision was normal, the patient being able to read No. 3 of Jaeger's types at twelve inches with the left, and No. 5 with the right eye. Examination of the fundi showed all the usual appearances of optic neuritis on both sides, most marked on the right, in the retina of which a number of small hæmorrhages were discernible. There was slight comparative immobility of the left side of the face, chiefly elicited by attempts at forced movements. The tongue when protruded pointed slightly to the left. Articulation was normal. The hearing was asserted by the patient to be normal, but was less acute in the right ear. A watch which on the left side was heard at three 
feet, was only detected on the right at eight inches. The common sensibility of the head, and the other special senses were normal. There was complete paralysis of the left fingers, thumb and hand. The movements of the elbowjoint were very limited, and those of the shoulder impaired. There was no attempt at supination or pronation of the forearm. There was no trace of rigidity or wasting of the muscles. The irritability to mechanical stimulus of those of the forearm was markedly increased, and the temperature of the skin was lower on the left as compared with the right side. The left lower extremity was stated to be weaker than the right, but, when the patient lay in bed, its movements seemed much the same as those of the other, but were performed with more hesitation and less alacrity. When walking there was slight lameness, the toes were not completely cleared from the ground, so as to necessitate slight swinging of the leg in progression. The limbs were of equal size and the muscles of normal appearance. Their mechanical irritability and the knee-jerk phenomenon were greater on the left side, though somewhat excessive in both. The temperature of both legs was equal. The sensibility of the skin was everywhere normal, and the appearance of both sides of the body was the same.

Progress of the case.-While under observation in the hospital the condition described continued. The patient suffered frequently from paroxysmal attacks of lancinating pains in the head. These lasted sometimes for twelve or more hours at a time, and they were so violent that the patient was occasionally delirious and kept the whole ward disturbed with his cries. There were intervals during which he was entirely free from pain. He also suffered from seizures of very severe sickness not specially associated with the headaches. During these he vomited all food, and when the stomach was empty continued to retch with great violence. This would sometimes last for several days, causing great distress, and much reducing the strength of the patient. During residence in the hospital 
the attacks of paroxysmal twitchings of the muscles were frequently observed. These occurred many times every day. The most common form was a rhythmical tremor which began in the first, second, and third fingers of the left hand, which afterwards spread to the thumb and wrist as far as the elbow. This continued for perhaps a minute, and then ceased, generally by the limb being held or rubbed. Another form began in the left angle of the mouth and side of the face, and a feeling as if the tongue was being contracted. These parts also continued to twitch for a minute or two. These two kinds of attacks rarely occurred at the same time, but took place independently of one another. Sometimes, but not commonly, the movements began in the face or arm, extended from the one to the other, and from thence down the side of the neck and body to the leg, so that the whole left side was convulsed without any loss of consciousness. The leg was never observed to be affected by itself.

Diagnosis.-The sequence of events described, with all the circumstances of the case, led to the diagnosis that there was an encephalic growth, probably of limited size, involving the cortex of the brain, and situated at the middle part of the fissure of Rolando.

Treatment.-The patient was ordered the bromide and iodide of potassium, twenty grains of each, thrice daily, which he took for a month. Ice to the head gave no relief, and the vomiting was unrelieved by any treatment. The severe pain was ameliorated by hypodermic injections of morphia.

The terrible sufferings of the patient rendered life intolerable to him. All remedial measures having failed, and as it was obvious that his symptoms were extending, and that a fatal termination was not far distant, it was determined that an attempt be made to remove the morbid lesion. It was hoped that even if such a proceeding was not permanently successful it might alleviate some of the more pressing symptoms. The novelty and risks of the proposed treatment having been fully placed before the 
patient and his friends, they readily consented to the adoption of any measures which offered any prospects of mitigating the urgent distress or of averting a certain death.

Operation.-In order to expose the cortex of the brain at the middle third of the fissure of Rolando the following procedures were adopted. A longitudinal line was drawn between the frontal and occipital protaberances, down the middle line of the scalp (Fig. 1, 1). A second line was drawn at right angles to this at the level of the anterior border of the external meatus of the ear (Fig. 1,2). Parallel to this a third line was drawn at the level of the posterior

Fig. 1.

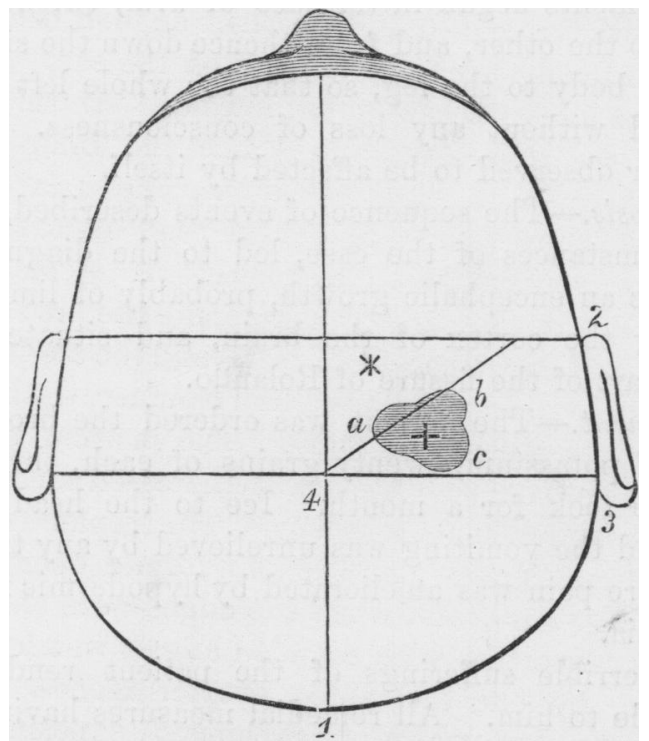

External surface of scalp. 1, 2, 3, 4. Lines to determine position of fissure of Rolando. + Theoretical and actual position of tumour. $*$ Tender spot on scalp. $a, b, c$. Position and order of trephine openings.

border of the mastoid process, which reached the longitudinal line about two inches behind the second (Fig. 1, 3). From the junction of the first and third lines, a fourth was 
drawn diagonally downwards, reaching the second at a point two inches above the external meatus (Fig. 1, 4). This diagonal line was believed to represent the direction of the fissure of Rolando. The spot where theoretically the centre of the trephine should have been placed was about half an inch behind the diagonal, and about one and a half inches from the longitudinal line (Fig. 1, +). As there was a tender point on the scalp about two inches anterior and to the inside of this (Fig. 1,*), it was determined to make the first opening in the skull between the two. (The order and position of the trephine openings are seen in Fig. 1, $a b c$.)

On November 25th, a trephine one inch in diameter was applied to this region (Fig. 1, $a$ ) and a circle of bone removed. The centre of the aperture was one and a quarter inches from the middle line and half an inch behind a line drawn vertically from the meatus of the ear. The dura mater was found normal in appearance. In this a crucial incision was made, through which the brain substance bulged, as was thought, abnormally. The surface appeared somewhat more yellow in colour than natural, but was otherwise apparently as in health. A second trephine opening was made, slightly overlapping the first (Fig. 1, b), external and slightly in front of it, and the angles thus left were rounded off with a chisel and hammer, the brain being protected by a copper spatula. The incision in the dura mater was prolonged, exposing an increased surface of brain but without further revelations. The trephine was applied a third time so as to join the two former openings posteriorly (Fig. 1,c), and when the edges were chipped off a triangular aperture with rounded angles was left, measuring two, by one and three quarter inches. The incision in the dura was then prolonged, exposing a surface of brain nearly the size of the opening in the skull, which presented the same appearance as that already described. Occupying most of this space and crossing it obliquely from above and behind, forwards and downwards, was a convolution, down the posterior aspect of which ran a large blood-vessel. Into the centre of, and parallel with 
this convolution, an incision about three quarters of an inch in length was made with a scalpel. From an eighth to a quarter of an inch below the surface, a transparent lobulated solid tumour was seen, thinly incapsulated, but perfectly isolated from the surrounding brain substance. After prolonging the incision in the cortex, the surface and sides of the growth were easily separated by means of a narrow spatula of steel so tempered that it could be bent into any shape required. The mass was conical in shape the base being upwards. After its superficial portion was isolated, the finger was, as far as possible, inserted behind the tumour, and attempts made to enucleate it. In doing so the upper half broke across. A large Volkmann's sharp spoon was then employed to scrape out the deeper parts of the growth; and this was continued till all the morbid material was removed and apparently healthy brain matter only remained. This part of the operation was rendered difficult by the rapid welling of blood into the wound. No artery of any size spouted but there was a general oozing, which accumulated rapidly as soon as the sponge was removed. The cavity thus left was about one and a half inches in depth and of a size into which a pigeon's egg would fit. The hæmorrhage was arrested by applying over the cut surface a suitable electrode from an electro-cautery. The dura mater was then drawn together at its anterior part by a few carbolised silk sutures, and a drainage-tube of moderate calibre, made of india rubber, was inserted into the wound beneath the dura at its posterior border. The skin was brought accurately together, except where the tube lay, by silver wire and silk sutures. During the entire operation the carbolic spray was used, and both before and after, all the ordinary antiseptic precautions were taken. ${ }^{1}$

1 The antiseptic precautions were as follows:-The patient's head having been previously shaved, except the very lowest purt of the scalp quite below the occiput, the whole was thoroughly soaked with carbolic acid lotion (1-20), but particular attention was not directed to the sores left from some blisters at the upper part of the neck, which were not noticed till afterwards. After the patient was placed on the table the parts were again washed with 1-20 lotion, and the upper portion of the body was surrounded by carbolised 
The wound was dressed with carbolic acid gauze, completely covering the scalp, and firmly fixed in position with bandages. During the entire operation, which lasted two hours, the patient took chloroform without a bad symptom, and no nervous phenomena were developed. Subsequent examination proved the tumour to be a glioma about the size of a walnut, presenting the usual microscopical appearances of that disease (Fig. 2).

Fre. 2.

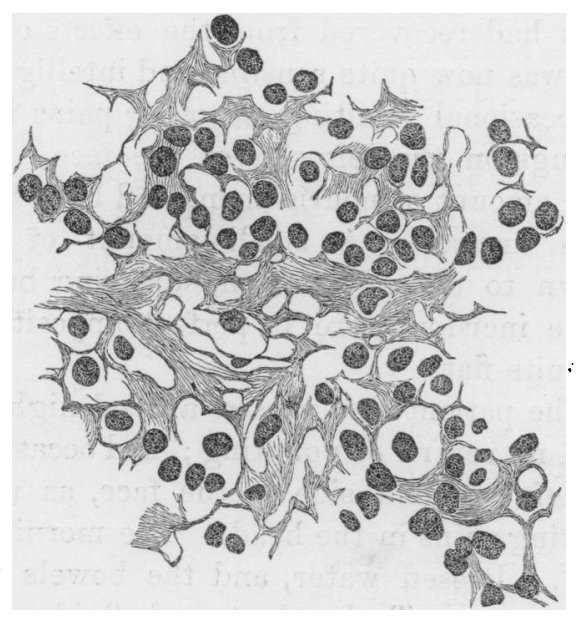

S. Structure of glioma, from section by Dr. Hebb, about 400 diam. :

Progress of the case after operation.-Half an hour after the operation the patient was in the usual drowsy condition which follows the administration of chloroform. He answered questions rationally and comprehended what was said to him. There was no increase of paralysis of the face or tongue, and articulation was as before. There was no. trace of movement in any part of the left upper extremity, but the left leg could be moved at will, but to what extent it was not judged advisable to determine. Six hours aftertowels, one being also placed beneath the head. Instruments and hands were soaked in the same lotion and the spray was used throughout the operation. 
wards the patient was quiet and comfortable. He had taken small quantities of milk and soda water, and had not been sick. Pulse 80, full and regular; temp. $98^{\circ}$. There was no pain in the head and only slight soreness of the wound was complained of. There was slight paresis of the left side of the face and occasional twitchings of both angles of the mouth, the cheeks, and the alm of the nose. There had been no movements elsewhere.

November 26th (the day after the operation).-Pulse 56, of good strength and regular, morning temp. 98.4\% The patient had recovered from the effects of the anæsthetic, and was now quite sensible and intelligent. There had been occasional vomiting, shooting pains in the head, and twitchings on the left side of the face. During the night a fair amount of nourishment had been taken. The wound was dressed. A small amount of serum had soaked down to the edge of the dressing behind. The edges of the incision were in perfect apposition, and the scalp was quite flat.

27th.-The patient had passed a good night, and slept well. Had no return of vomiting; had occasional twitching of the left arm and side of the face, as well as occasional shooting pains in the head. The morning pulse 54, temp. 97.6 ${ }^{\circ}$ Passed water, and the bowels acted freely after an enema. Took plenty of fluid nourishment. The patient was quiet, and at time of visit without pain. He was somewhat drowsy and apathetic, but perfectly intelligent, and answered questions with promptitude and accuracy. Articulation was normal. The movements of the eyeballs were natural. The pupils were somewhat dilated, equal, and contracted to light. There was distinct but slight paresis of the left side of the face. The tongue, when protruded, inclined somewhat to the left. The left upper extremity was throughout immovable. The left leg could be raised and pulled up when desired, but for fear of disturbing the patient, the amount of this was not fully tested. The left knee-jerk was markedly increased. The sensibility of the skin was everywhere 
normal. There had been nowhere any twitchings for the last twenty-four hours. During the day there were occasional shooting pains in the head. The wound was left untouched as there was no appearance of discharge.

28th.-During last night the patient slept fairly well, but was occasionally disturbed by sharp pains in the head. Morning pulse 60 , temp. $98 \cdot 6^{\circ}$. The patient was bright and cheerful. He has had no twitchings of the face or limbs, but had suffered from occasional pains in the head. The paresis of the face was very slight, the articulation natural, and the condition of the limbs as before. He had taken plenty of nourishment. When the wound was dressed the discharge was found to consist of a small amount of thickish pink serum, with an obvious though faint smell. There was a distinct bulging of the scalp at the seat of operation, where it was slightly tender.

29th.-The patient had passed a good night. Morning temp. $98.6^{\circ}$, pulse 76 . During the day there was no return of the pains in the head, or twitchings of the limbs. The general condition was as before. The patient was in good spirits, intelligent, and took food well. The left leg could be moved at will. During the evening there was some swelling of the eyelids and face, accompanied with smarting pain. On changing the dressings the lips of the wound was found swollen, and the discharge had a decidedly putrefactive smell. The lower lateral incision was opened up, and the drainage-tube removed and washed. From the openings some thick brown pus was squeezed. The scalp in the neighbourhood of the wound was somewhat cedematous. Dressings were reapplied as before.

30th.-The general condition was as before, except that to-day there were no movements of the left lower extremity. Morning temp. $99 \cdot 4^{\circ}$, pulse 88 . The swelling of the face and scalp was very considerable. In the morning the drainage-tube and several stitches were removed, and a dressing of wet boracic lint substituted for the gauze. The wound was freely syringed with carbolic 
lotion (1-20) in which jodoform was suspended. The dressings were changed twice during the day, and on the last occasion all the stitches were removed. By this time there was hardly any trace of putrefactive smell. A hernia cerebri as large as half an orange, consisting of granular looking matter mixed with blood-clot, had protruded through the lips of the wound. Towards evening the swelling of the face and scalp had considerably diminished. At midnight the patient felt quite comfortable.

December 1st.-To-day an extended examination was made. Morning temp. $98.4^{\circ}$, pulse 88 . The patient felt well in every respect. He was cheerful, perfectly intelligent, conversed freely, and took an interest in the details of his case. For some time there had been no trace of twitchings of the limbs or pains in the head. The swelling of the face and scalp had almost disappeared. The pupils were equal and normal. Vision was normal. The movement of the eyeballs was natural. The skin was cool and moist. The mouth and face were drawn slightly to the right on forced movement, and the tongue was protruded slightly to the left ; articulation and hearing were as before. There was no movement whatever of any part of the left upper or lower extremities. The sensibility of the skin of the left limbs was considerably diminished to touch, but not lost. The left knee-jerk was still considerably exaggerated as compared to the right. The plantar reflex was the same on both sides. The general condition of the patient was excellent, and his appetite good. The urine was loaded with urates, but otherwise normal. The wound was dressed morning and evening. The hernia, which had somewhat increased in size, was freely treated with carbolic acid, iodoform, and a solution of chloride of zinc (gr. $x l-3 j$ ). The discharge had almost lost its offensive smell.

2nd.-The general condition as before. Now there was no trace of cedema of the face or scalp. Morning temp. $98.6^{\circ}$, pulse 88 . The hernia cerebri appeared to be somewhat larger. 
3rd.-The general condition as before. Morning temp. $97 \cdot 6^{\circ}$, pulse 54 . The greater portion of the hernia, which had now reached the size of half a cricketball, was clipped away with scissors. The parts removed consisted chiefly of granular matter and blood-clot, and apparently contained little true cerebral matter. They had a faint offensive smell. This removal was continued till a surface was reached where the tissues bled freely, which was only very slightly above the level of the scalp. This was freely treated with a strong solution of chloride of zinc and iodoform. The stump was dressed with boracic lint soaked in carbolic lotion, which was tucked under the edges of the flaps. The whole as at former dressings was enveloped in a mass of salicylic wool and firmly bandaged. Immediately after the dressing the temperature was $100^{\circ}$. In the evening when the wound was dressed again the discharge was found copious and watery.

4th.-The general condition as before. Morning temp. $98 \cdot 4^{\circ}$, pulse 88. A cap of block tin was fitted over the hernia which bled slightly from small points all over its surface. The discharge was diminished and now quite odourless.

13th.-During the last ten days the general condition of the patient had continued to improve. On examination to-day he felt well in every respect. There had been no pain in the head or twitchings in the face or limbs. The appetite was excellent and all the organic functions were normally performed. The disposition was cheerful and the intelligence perfect. The patient conversed all day with the nurse, who could detect no signs of mental failure. There was still slight paresis of the left side of the face and tongue. The movements of the eyeballs were normal. The pupils were equal, and contracted normally to light and accommodation. Vision was apparently normal, and No. 7 of Jaeger's types could be read with the right and No. 4 with the left eye at twelve inches in rather a bad light. The optic neuritis still existed in both eyes, but was distinctly improved, the 
hæmorrhages in the right having almost completely disappeared, and the swelling of the left disc being almost gone. The sensibility of the face was normal and all the special senses were as before the operation. The entire left upper extremity still remained completely paralysed, the muscles being limp and flaccid and without trace of rigidity. The left lower limb was also without voluntary movement. The sensibility of the skin of the entire left side, below the neck, was diminished to touch, but not altogether lost. The temperature of both sides was nearly the same. The mechanical irritability of the muscles and the tendon-reflexes on the left side were increased as compared to those on the right, and on the left there was well-marked ankle clonus. There was nowhere any rigidity or wasting of the muscles.

During the last ten days there was no essential change in the wound, except from day to day a gradual increase in size of the hernia. This now projected about an inch above the scalp. Its surface was smooth and clean, and the discharge was copious, colourless, and odourless. The margins of the mass were clipped away so that no mechanical obstruction might be offered to the contraction of the flaps, which process seemed to be in progress. In doing so a vascular part was soon reached, and a clear fluid in considerable quantity exuded. The deep surface of the flaps was covered with healthy granulations.

16th.-The patient continued well till last night, when he was restless, and felt his left arm and leg very cold. About six this morning he was seized with a rigor which lasted five minutes. Soon afterwards he suffered from shooting pains in the head and was very sick. Five hours after, the temperature was $100.2^{\circ}$, the pulse 100. The patient complained of severe pain in the frontal region. He was dull, apathetic, and nauseated. There were twitchings in the right arm and leg, and occasionally slight ones on both sides of the face. Until to-day there had been little change in the wound, except 
that the flaps were gradually drawing together and tending to cut off the superficial part of the hernia. This morning it was found that the hernial mass had greatly increased in size. It was now rugged and of a dark colour. Attempts to remove portions of it were prevented by the profuse hæmorrhage. There was no putrefactive smell. In the evening it was found that the patient during the day had suffered much from pain in the head and vomiting. A hypodermic injection of morphia had been given in the afternoon, which had produced sleep for several hours. The temp. was $102 \cdot 2^{\circ}$, the pulse 100 . Later the temp. was $104 \cdot 6^{\circ}$, the pulse 140 .

17th.-Morning temp. $102 \cdot 6^{\circ}$, pulse 140. Passed a very restless night, suffering much pain in the head, and in the right arm and leg. Was found pale and dull and very feverish. The patient understood what was said to him, but was slow to answer, and his replies were not easily understood. Another injection of morphia was given without inducing sleep or relieving pain. The hernia was found to have greatly increased in size, and was again about the size of half a cricket ball.

18th.-The patient passed a fairly quiet night. Morning temp. $101 \cdot 2^{\circ}$, pulse 140 . Has had little or no pain in the head, was quite intelligent and answered questions sensibly. There were no twitchings or increase of paralysis. He was feverish and very thirsty, but continued to take a good amount of fluid nourishment. The hernia was breaking down by a sloughing process, and a considerable quantity of semi-fluid detritus flowed from the cavities forming in the mass. This had again a slight putrefactive smell.

19th.-The general condition was the same as yesterday. The hernia was clipped off almost to a level with the bone, and a flat plate was placed and secured over the stump. In the evening the patient was very feverish and very talkative. He volubly related incidents in his past life, and carried on conversations quite sensibuly with imaginary persons. He was very

vOL. IXVIII. 
restless and had no sleep. He was intelligent and answered questions correctly. Articulation was somewhat thick and indistinct. There was no apparent increase of paralysis, and the right limbs moved freely as before. He still continued to take plenty of nourishment. Evening temp. $103 \cdot 6^{\circ}$, pulse 150 .

20th.-Has had no sleep for twenty-four hours.

Was still very restless and feverish, but had no pain. Was evidently weaker, but there were no new symptoms. Temp. 104 ${ }^{\circ}$, pulse 150. When the eyelids were opened both eyes were seen deviating to the left, but could be voluntarily fixed in a straight line. No change in the wound.

21st.-The patient was evidently sinking. He was emaciated and a bedsore had developed on the right gluteal region. Still feverish, restless, and sleepless. $\mathrm{He}$ continued to talk volubly with a thick indistinct utterance. Temp. $104 \cdot 4^{\circ}$, pulse 144, very weak. Was perfectly sensible. Bowels relaxed and motions passed involuntarily in bed. There was a general tremor of all the limbs, and the right side occasionally twitched.

22nd.-Had gradually become weaker. The breath had a sweatish smell and the skin a yellowish waxy appearance. The articulation was so indistinct as to be unintelligible. Had no pain, no sleep, was very feverish, and now refused food. Was still perfectly sensible.

23rd.-Since last report gradually sank, and died at 8 a.m. this morning. No new symptoms occurred and the patient was sensible to the end. The condition of the wound continued as last described.

The post-mortem examination (December 24th, thirty hours after death).-The body throughout was thin but not greatly emaciated. There was no special muscular wasting, the rigor mortis was well marked, and the skin was everywhere of a pale yellow colour and of waxy appearance. On the most prominent part of the left gluteal region was a circular patch three inches in diameter, of black discolouration. On cutting across and into this, it was 
found to extend an inch in depth into the tissues, including a portion of muscle. On the right parietal region was an open wound of the scalp. This was of an irregular quadrilateral shape and measured three by two and a half inches. It reached to within half an inch of the middle line of the skull and its direction in its longest axis lay between this and the posterior margin of the ear. The edges were somewhat raised, everted, covered with healthy granulations, and for a quarter of an inch free, beyond which the skin was adherent to the bone. Elsewhere the scalp was healthy, and there were no signs of pus or putrefactive smell. The space between the edges of the wound was filled up by the base of the hernia which had been shaved off. This spread over the surface of the bone to which it was adherent, and had to be cut away with the knife. The removal of this exposed the aperture in the skull made by the trephine. 'This was of triangular shape with blunt rounded angles and measured two, by one and three quarter inches. Its longer axis lay almost exactly between the parietal protuberance and the central line of the skull, reaching to within about half an inch of both. The edge of the bone was perfectly healthy and presented the appearance of a clean cut. The skull cap was removed in the usual manner and the brain and cord were taken out, when it was seen that the inner aspect of the arachnoid at the base of the cranium was lined by a layer of pale yellow, coagulated, recent lymph. This was most abundant in the right middle fossa and over the base of the sphenoid bone. It also extended for a short way down the spinal canal. The dura mater of the base was somewhat thickened but otherwise normal.

The brain.-On inspecting the base of the brain a thin layer of lymph was found spread over the surface of the arachnoid. This was most abundant over the base of the right temporo-sphenoidal lobe, over the pons and medulla, and down the upper part of the spinal cord for about an inch. It reached forwards, but less in amount, to the bases of the frontal lobes, sideways to the inner 
edge of the left temporo-sphenoidal lobe, and backwards as far as the anterior border of the cerebellum. The outer edges of the cerebellum, most of the left temporosphenoidal lobe, and the under surface of the frontal lobes were entirely free from the effusion. This deposit of lymph extended from the base of the brain over the right temporo-sphenoidal lobe, and could then be followed by a tract about an inch wide to the under surface of the wound, from which it evidently emanated. The membranes covering the under surface of the right temporosphenoidal lobe were markedly congested and their vessels dilated. There was also slight injection of those at the under surface of the cerebellum. Elsewhere the membranes of the base were healthy. The convolutions of the base of the brain were everywhere normal in appearance, except that those of the right temporo-sphenoidal lobe were somewhat more flat and the sulci less deep than those of the opposite side. The consistency of the cortex was here everywhere intact, except a patch of slight softening about the size of a sixpenny piece at the most anterior extremity of the temporo-sphenoidal lobe. The external surface of the dura mater covering the hemispheres was on both sides normal in appearance, except over the right parietal region, where the wound had been made, through which protruded the base of the hernia cerebri. This corresponded almost exactly in size, shape, and position with the aperture in the skull and measured two and a half by two inches. The free edge of the dura was adherent all round to the cut surface of the bone. On reflecting this from the brain it was found normal in every respect on the left side. On the right it was somewhat thickened throughout, and very considerably so immediately round the wound, especially behind and below. Here also in several places there were recent adhesions of the two layers of the arachnoid which were readily torn across without force. Traces of lymph were found scattered over the whole hemisphere, but chiefly over the occipital and temporo-sphenoidal lobes. It was most abundant in 
a narrow tract stretching from the lower border of the wound, proceeding downwards to the base of the brain as already described. Above and in front of the wound there was no appearance of inflammatory exudation. Throughout the left side there was slight subarachnoid effusion. The anterior lobes were pale and normal in colour. Behind a vertical line drawn through the bases of the frontal convolutions the membranes at the upper part of the cerebrum were of a pinker colour, and their vessels more injected with blood, than in front and below; otherwise their appearance was normal. The convolutions on this side appeared to be slightly flattened and the sulci somewhat shallow, otherwise they were normal. On the right side the membranes above and in front of the wound were precisely the same as on the left. Behind and below, and especially over the superior part of the temporo-sphenoidal convolutions, they were intensely congested. The convolutions of the frontal and parietal regions on this side, both in appearance and consistency, were in all respects the same as those on the other, and they were equally voluminous on both sides, but the parietal area had a shrunken appearance as if it had fallen inwards. In the centre of this, and occupying the position of the fissure of Rolando, was the wound in the brain. It corresponded in position to the hole in the skull, but was a trifle larger, measuring two and a half by two inches, the longest axis being directed somewhat obliquely from above downwards.

The destruction of the cerebral cortex is illustrated in the accompanying diagrams (Figs. 3 and 4), and will be seen to involve, first, the entire length and thickness of the ascending parietal convolution with the exception of a small portion of its superior and inferior extremities, both of which remained intact; secondly, almost the entire upper third of the ascending frontal convolution, and the posterior portion of its upper half ; and thirdly, the anterior third of the gyrus supramarginalis. This deficiency in the grey matter was occupied by the rough material 
constituting the stump of the hernia cerebri which projected about half an inch beyond the surface of the brain. Surrounding and closely adjacent to this on its anterior, superior, and posterior aspects, the cerebral cortex was normal in appearance and firm in consistency. The margin of the aperture in the grey matter was sharply cut, slightly folded inwards, and its inner edges were adherent to the hernia. At the inferior border of the wound the convolutions, although normal in appearance, were

\section{Fig. 3.}

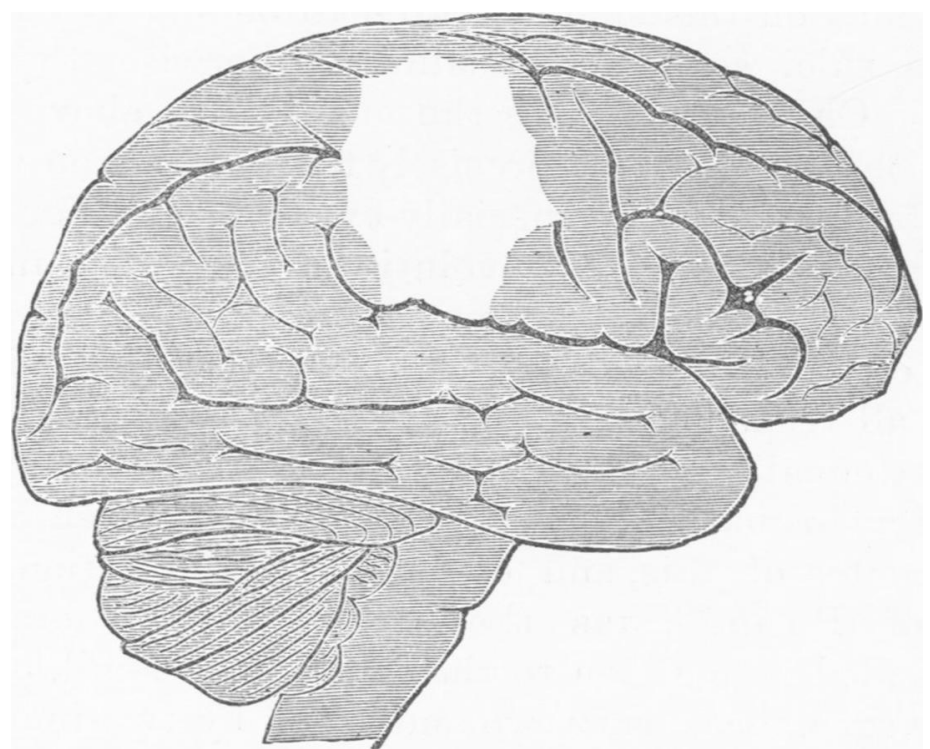

Diagram showing position and extent of cerebral cortex destroyed as seen from without. The white space occupied by stump of hernia cerebri.

slightly softened to the touch, and this softening extended so as to include the superior temporo-sphenoidal convolution, below which the cortex was of firm and normal consistence. On the inner aspect of the right hemisphere there was a circular depression, about the size of a sixpenny piece, without softening, and which appeared as if the part had fallen inwards. This involved the terminal purtion of the fissure of Rolando, and a part of the sulcus caloso-marginalis, a small portion of the 
gyrus fornicatus, the posterior part of the marginal convolution, and the anterior border of the quadrilateral lobule. A transverse section was made across the brain through the ascending parietal convolution in the direction of the fissure of Rolando. The deficiency in the cortex is seen in Fig. 4 to consist of complete absence of that part which corresponds to the middle parietal fasciculus with the inferior portion of the superior, and the superior portion

FIG. 4.

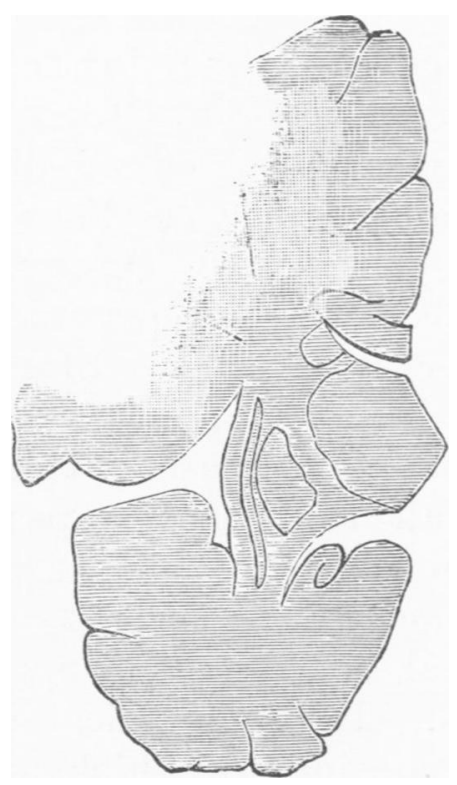

Diagram of transverse section through ascending parietal convolution showing destruction of cerebral cortex and corona radiata. The white space occupied by granular matter of hernia cerebri.

of the inferior parietal fasciculi. The spaces thus left had partially collapsed, and were filled with new formation from which the hernia cerebri sprang. This morbid condition had in an irregular and unequal manner spread inwards, reaching as far as the upper margins of the corpus callosum and internal capsule. The brain having undergone the process of hardening it was not easy to exactly limit the softening, which seemed to be confined entirely to the centrum ovale. The lower portion of the 
inferior, and the inner portion of the superior parietal fasciculi were uninjured. So also were the insular lobe, the internal and external capsules, the corpus callosum, the optic thalamus, and the lenticular and caudate nuclei. Whether the lateral ventricle had been opened into it is difficult to say. There was no collection of foreign matter in its interior ; at the same time the morbid process had extended in close proximity to it.

A histological examination of the different tissues was made by Dr. Hebb, who reported their condition to be as follows :

"The appearance of a section of the glioma has already been referred to (Fig. 2). The hernia cerebri and subjacent tissues consisted of blood-clot, granular matter, and disorganised nervous tissue. The cortex in the neighbourhood of the wound presented the appearance of inflammatory change in its pia mater and superficial layer, but was otherwise normal. Elsewhere the structure of the brain was healthy, and nowhere was there any evidence of gliomatous disease. The retinæ and optic nerves presented all the usual appearances of neuroretinitis in a well marked but not advanced stage. The membranes of the cord had undergone marked change. The inter-meningeal space at the upper cervical region was filled with pus. In the cervical and dorsal regions the membrane was considerably thickened, which condition gradually diminished from above downwards. In the grey matter of the cord there was evidence of nuclear activity, apparently of quite recent origin, otherwise it was healthy. There was no trace of descending sclerosis."

Commentary. - In commenting on the preceding series of facts it will be convenient to discuss the points of interest under the following heads : (1) Diagnosis, (2) the surgical treatment, (3) the clinical phenomena after the operation, (4) revelations of the autopsy, physiologically and pathologically considered.

1. Diagnosis. - All the circumstances of this case pointed to an encephalic growth on the right side. This was 
more especially evidenced by the slow and insidious invasion of the symptoms, the gradual progress and special distribution of the paralysis, the violent intracranial pain, the uncontrollable attacks of vomiting, and the double optic neuritis. Whether a tumour on the right side of the brain was caused by the blow on the left side of the head a year previous to the development of the first signs of ill-health, must remain uncertain.

It was also concluded that the morbid lesion involved the cortical substance, because certain motor phenomena were developed, and certain motor districts implicated after a definite method and in definite order; the paralysis was unaccompanied by any alterations in sensibility ; and above all, because of the existence of certain paroxysmal seizures of local convulsion, without loss of consciousness, which were eminently suggestive of irritation of cerebral grey matter.

The special seat of the lesion was further believed to be the middle part of the right fissure of Rolando. This conclusion was arrived at by the consideration of the combined revelations of physiological experiment and clinical observation. After centuries of doubt and confusion on the subject of cerebral localisation, quite recent investigations have at last rendered it certain that around this sulcus are grouped those nervous areas which preside over the movements of the other side of the body. Adopting the topography of the brain constructed by Professor Ferrier as the result of his well-known researches, it may be said in general terms, that the motor centres which govern the voluntary movements of the lips and tongue are situated in the lower portions of the ascending parietal and frontal convolutions. Higher up in the same gyri are the areas for the muscles of the face. Occupying the middle portion and nearly the whole extent of the ascending parietal convolution are the centres of the fingers and hand. In the middle of the ascending frontal convolution are those elements which originate movements of the arm and upper arm, including flexion, pronation, and supina- 
tion of the forearm. At the superior and posterior aspect of the ascending parietal convolution is the centre for the lower extremity, and at the upper and anterior portion of the ascending frontal convolution are centres for complex movements of both the upper and lower limbs. Now, in the case before us there was complete paralysis of the fingers and hand, with inability to pronate and supinate the forearm, there was partial paresis of the movements of the elbow, and weakness of those of the shoulder-joint. There was also slight paresis of the leg and one side of the face. Accompanying all these there were paroxysmal convulsions in all these regions, occurring either singly or in definite order one after the other. These phenomena were to be accounted for by an extensive but not absolutely complete destruction of the motor centres of the fingers, hand, and forearm, with slight encroachment on, and irritation of, those of the face, upper arm, and leg. A very definite localisation of the disease was thus permitted, and the tumour was pronounced to have occupied the whole thickness of the middle two fourths of the ascending parietal convolution, and a portion of the adjoining upper half of the ascending frontal convolution. The morbid lesion, whilst almost completely destroying these areas, in addition modified the functions and caused irritation of those in their neighbourhood, without seriously involving their structure, namely, the siperior and inferior extremities of the ascending parietal and frontal convolutions, and the postero-parietal lobule.

Having thus accurately localised the position of the tumour its size could as a consequence be approximated. Assuming the disease to be limited to the cortex at the point already indicated, the fact that the centres of the leg above, of the face and tongue below, of the sense of sight behind, and of the movements of the eyeballs in front, were not seriously involved, proved that the growth was of limited size. A glance at the relative position and size of the convolutions of the human cerebrum indicate that a foreign body occupying such a position could not, roughly speaking, 
exceed some two inches in diameter. It was probable that the growth took its origin in the lower third of the ascending parietal convolution, and as it increased in size spread upwards and backwards, further involving its substance and part of the ascending frontal convolution, finally reaching the lower edge of the postero-parietal lobule. Such disease might therefore be represented by an ovoid mass the size and shape of a pigeon's egg, lying obliquely in the fissure of Rolando. This theoretical reasoning arrived at before the operation, subsequently proved to have been substantially correct.

The question finally arose whether the tumour was confined to the cortex or whether it was situated in the centrum ovale below, and from thence invaded the grey matter. It was not forgotten that a slowly-growing mass, reaching considerable dimensions, might develop in the conducting elements of the brain, without causing symptoms capable of exact definition. It was also fully recognised that a small tumour immediately under the cortex and involving its under surface might cause precisely the same symptoms as one limited to the grey matter. From an operative point of view the existence of even a large growth, which in this case was improbable, would not necessarily interfere with the procedure, because in that case little harm could be done to the life of the patient, and his urgent symptoms, on the other hand, might be relieved by the simple process of trephining. If the tumour was small the grey matter could be incised and the mass removed. As a matter of fact this last is what actually was done in the case under consideration, the growth being found in the centrum ovale, under the cortex, involving the convolutions before determined.

As to the probable nature of the tumour, the age of the patient, the absence of syphilis, and the slow growth of the disease suggested glioma, but on this point no definite conclusions were hazarded.

2. The surgical treatment.-For the purpose of removing the tumour from the brain, three trephine holes 
were made in the skall, because after the first piece of bone was taken away and no superficial disease discovered, it was thought advisable to enlarge the opening, to further expose the brain and to make room for completing the operation. The aperture made by the three removals, even when the angles were chipped off, only measured $2 \times 1 \frac{3}{4}$ inches. The tumour was found exactly in the centre of the opening, and in the position corresponding with theoretical calculation and measurement. It had no relation whatever to the tender spot on the skull, and, had attention been confined exclusively to that, the result would have been entirely misleading. The process of trephining involved no difficulties, but it exemplified the advantages of the chisel and hammer over Hay's saw for rounding off corners of bone. In similar operations it might be advisable in the future to employ a larger trephine. One convolution only being exposed during the operation, there was at the time some question as to whether it was the ascending frontal or parietal. This doubt arose from the circumstance that in the attempt to approach the tender spot, the theoretical position had been slightly departed from, and the trephine advanced and raised, so as to come between the two. Accordingly for the moment this convolution was thought to be the ascending frontal, the aperture in the bone being so small that its relations could not be seen. After death, however, it was apparent that the convolution which had been incised was that in which from the first the disease had been diagnosed to exist, namely, the ascending parietal. There was no external appearance of disease about this part of the cortex except that it seemed swollen, less glossy, and less vascular than natural. An incision into this showed the morbid growth to be immediately under the surface, and almost completely involving the entire thickness of the cortex. On clearing the superficial parts of the growth, a small spatula, neither sharp nor blunt, and so tempered that it would keep any shape given it, was found a most serviceable instrument. Such is preferable to the 
use of the cautery, as the latter so chars the parts as to modify their natural appearance, and thus prevent a differentiation between bealthy and diseased tissues. It may be questioned whether it was advisable to arrest the hæmorrhage from the interior of the wound by means of the galvano-cautery. Such a proceeding leads of necessity to the formation of a considerable amount of detritus which may afterwards prove detrimental. The bleeding moreover was not severe and would no doubt have become arrested by natural means. The advisability of introducing a drainage-tube may also be questioned. It was not judged safe to completely close so large a wound, distended as it must have been with accumulated serum and blood. Moreover, had putrefaction not occurred it is not likely that the soft india-rubber tube would have caused any serious irritation. The argument in favour of complete closure of the wound, so strongly advocated by those whose experience is confined to operations on the brains of monkeys, is, it is maintained, not convincing when applied to a large injury in the human subject, the more rapid healing of the tissues of the lower animals being a matter of common knowledge. Another point of doubt is the propriety of introducing sutures into the dura mater. The most important matter for discussion, however, is the occurrence of putrefaction, which undoubtedly appeared in the wound some days after the operation. This, it may be maintained, was the cause of the inflammation and consequent hernia cerebri. All the usual antiseptic precautions were taken during the operation, and the only flaws in its strict application which suggest themselves are, first, that the scalp was not sufficiently purified prior to the operation, and second, that no special measures were taken to carbolise the galvanocautery apparatus. In future operations of this nature it is strongly urged that surgeons should not only employ carbolic acid, but also a solution of corrosive sublimate as antiseptics, and that the scalp should not only be rubbed with these, but soaked with them for some hours previously. 
There may have been other sources of septic contagion in the washing of the sponges, or from the blisters on the neck which escaped observation. It may be doubted whether the putrefaction was ever completely subdued; the fact of the meningitis occurring at last, and that of a smell having again become apparent after the attempt at removal of the second protrusion, point probably to a continued septic infection. As to the hernia two observations only have to be made. First, it was remarkable that the discharge continued for such a long time to be so copious and so watery, which suggested the idea of its being cerebro-spinal fluid. Secondly, there was a difficulty in shaving it off owing to the enormous size of its base, and to the danger of serious hæmorrhage.

3. Clinical phenomena following the operation.-The patient, on recovery from the effects of chloroform after the operation, was found perfectly intelligent, the former pain in the head, and violent twitchings in the limbs, had disappeared and never returned, there was no increase of the paralysis of the face or leg, and all the organic functions remained normal. The only change which had taken place was completion of the paresis of the upper extremity, which was now paralysed throughout. This was evidently due to the unavoidable destruction of the remaining arm centres in the removal of the tumour. Otherwise the neighbouring brain matter had not been injured, as was evidenced by all other functions remaining intact. The surgical operation itself in no way injured the nervous centres with the exceptions mentioned, while it immediately relieved all the distressing symptoms. This satisfactory condition remained unchanged for four days, when the discharge from the wound was found to have a putrid smell. Coincident with this began the hernia cerebri, and following its development, arose fresh symptoms in the shape of paresis of the left leg and partial anæsthesia of one half of the body. These were probably due to the effects of simple pressure, and possibly to the subsequent secondary softening of the conducting 
fibres caused by it. That the inflammatory condition which led to this was purely local was shown by the fact that, with the above exception, the condition of the patient remained in all respects as before the operation. The temperature never reached $100^{\circ}$ or the pulse 100 beats per minute. The intelligence was absolutely intact and the appetite and general condition in every respect satisfactory. The patient had lost all pains in his head, all traces of twitchings of the limbs, and all his severe attacks of vomiting. Even the double optic neuritis had markedly diminished. This state continued daily to improve till the twenty-first day, when suddenly the patient was seized with a rigor followed by fever and all the symptoms of meningitis from which he died a week afterwards. This inflammation was afterwards seen to be local and due to septic matter from the wound causing irritation of certain areas of the cerebral membranes. If putrefaction was the sole cause of this condition, hope may be entertained that by its prevention in other cases a more satisfactory termination may be looked for. Although meningitis continued to a fatal end, no new nervous symptoms supervened, the absence of which was probably due to the presence of a hole in the skull, through which excess of pressure was relieved.

4. Revelations of the autopsy.-After death, inspection of the parts showed that the brain was practically everywhere healthy except the area injured by the operation and the membranes in its immediate neighbourhood. From its lower border a narrow tract of recently effused lymph extended downwards by the temporo-sphenoidal lobe towards the base of the skull, over a large portion of which it spread, leaving the adjacent parts healthy. It was therefore obvious that this condition was produced by irritating matter from the interior of the wound, flowing downwards between the layers of the arachnoid, accumulating at the base, and by its presence causing meningitis in its track. The local inflammation of the wound had so opened out the parts and separated the adhesions as to permit the 
discharge to percolate into the cranial carity, but not till three weeks after the operation. Had this not occurred there is no reason why the healing process should not have been maintained, and the entire wound become ultimately cicatrised. The patient would then have continued permanently in a satisfactory condition, and escaped the secondary and fatal complication. The recovery from serious surgical injuries to the brain-substance of man, as well as experimental researches on that of animals, show that such a termination is perfectly possible.

The cortical substance at the edges of the wound in the brain was firm and healthy, except at the inferior border, which was slightly softened, probably from infiltration of the meningeal effusion. The deficiency in the grey matter was clearly defined and the portions of absent convolutions could be accurately limited. On the subject of central localisation only general conclusions can be drawn, as the destruction was not limited to the cortex, but in great part was situated in the centrum ovale below. The fibres, however, thus injured were those corresponding to the grey matter above, and may therefore be said to represent the conducting media of the higher centres. The symptoms immediately before the death of the patient, as far as they go, entirely harmonise with those which have already been determined by experimental inquiry to arise from corresponding lesions of cortical matter, with others superadded, which can be easily explained by the processes of pressure and softening in the neighbourhood. The inferior extremities of the ascending frontal and parietal convolutions being found only very slightly. involved, accounts for the almost total absence of orolingual symptoms during life. The almost total destruction of the remainder of the ascending parietal convolution explains the complete paralysis of the fingers and hand, and the partial paresis of the face. The lesion of the middle third of the ascending frontal convolution produced the immobility of the elbow- and shoulder-joints, and the loss of pronation and supination in the forearm. The 
almost complete immunity from disease of the lower part of this gyrus permitted the nearly natural movements of the face, lips, and tongue during life. The bases of the three frontal convolutions were perfectly healthy, but a day or two before death temporary conjugate deviation of the eyeballs was observed, both being turned towards the left, which was probably due to irritation of these regions by the neighbouring disease. At no time was there any paralysis of the muscles of the eyeballs. The posteroparietal lobule was found almost intact, its anterior margin only being involved in the wound. For some days after the operation the patient moved his left leg freely, and it was only after the appearance of the hernia that the limb became paralysed. This was therefore due not to destruction of the cortical centre of the lower extremity, but to pressure and softening within the wound. This was evidenced by the sinking in of the healthy convolutions on the inner aspect of the hemisphere at a point exactly corresponding with the situation of the conducting fibres of this region. The anterior portion of the supra-marginal gyrus was absent. This convolution Professor Ferrier associates with the sense of sight. In this case there was no evidence of any serious impairment of vision or hemiopia, although the patient saw better with the left than with the right eye. There was, however, double optic neuritis, most marked on the right side. The deficiency in sight was evidently due to this and not to a central lesion, in which case the weakness of vision would have been chiefly in the opposite or left eye. It is therefore probable that no appreciable loss of function could be attributed to the disorganisation of a portion of the right supra-marginal gyrus. It is, however, to be observed that the convolution was only partially destroyed, and Professor Ferrier has shown that even when it is completely obliterated on one side the consequent blindness on the other is only temporary, the opposite centre apparently rapidly compensating for the loss. Shortly before death the patient, though sensible, talked very volubly, carried on vor. LXvirr. 
conversation with imaginary persons, and recited the most elaborate and yet perfectly coherent adventures. May these not have been the result of visual hallucinations, and due to irritation of this centre ? ${ }^{1}$

Although the right superior temporo-sphenoidal convolution was somewhat softened it was not so to any great extent, and it was probably recent and due to mechanical infiltration. During life the hearing of the left ear was perfect. The comparative deafness on the right side was due to deficiency in the auditory apparatus and not to a central lesion.

The destruction of the centrum ovale for the main part corresponded with that of the cortical substance above. Its exact limits were difficult to define owing to the gradual softening in the neighbourhood. The internal capsule, corpus callosum and basal ganglia were, however, intact. So also was the remainder of the brain. The intellect, other senses, with all the organs and functions of the body except those already detailed, remained normal till the last.

Such are the main points of interest and reflections concerning a case which throughout has been a source of great anxiety and responsibility. This has chiefly been due to the fact that we have not had the advantage of any precedent of a like nature to guide us in our methods of procedure. Operations on the brain-substance have not been uncommon in the history of medicine, but these have hitherto been performed either for the relief of surgical injuries, or for disease indicated by local manifestations. We have nowhere been able to discover the recorded example of a case where a cerebral tumour was diagnosed by the symptoms observed, without visible or tangible external signs, and was in consequence operated on and successfully removed. Since this has been accomplished in the present instance, the public papers have asserted that the same has already been carried out on

1 In connection with the sense of sight, the fact may be noted that the optic neuritis was most severe on the side of the cerebral lesion. 
several occasions in the Royal Infirmary of Glasgow. To this it can only be said that up to the present date no report of such proceedings is to be found in medical or scientific literature.

In conclusion, we would observe that, although unfortunately in this instance life was not permanently preserved, the experience we have gained by this case leads us to the belief that there is an encouraging prospect for the future of cerebral medicine and surgery, and that as a tumour of the brain can be diagnosed with precision and successfully removed without immediate danger to life, we confidently anticipate that under more favorable circumstances the operation will be performed with lasting benefit to the patient.

(For report of the discussion on this paper, see 'Proceedings of the Royal Medical and Chirurgical Society;' New Series, vol. i, p. 438.) 